Methods 36 Wistar rats were divided randomly into three groups: control group ( $n=12)$, angioplasty group $(n=12)$ and Bosentan (BA) with angioplasty group $(n=12)$. Bosentan was administrated del to rats of BA with angioplasty group. The balloon catheter injury was performed on left common carotid artery of rat by imitating the process of angioplasty. After 7 days and 14 days, neointimal area (NIA) and stenosis index (SI) were calculated. Prior to angioplasty and 7 days after balloon injury, serum VEGF were assessed. In another experiment, 65 Wistar rats were divided randomly into three groups: control group, angioplasty group and Bosentan with angioplasty group. Bosentan was administrated to rats of Bosentan (BA) with angioplasty group. The balloon catheter injury was performed on left common carotid artery of rat by imitating the process of angioplasty. The process of neointimal and media hyperplasia was observed and $\alpha$-action and PCNA expressions were determined.

Results NIA and SI of BA with angioplasty were significantly decreased compared with angioplasty group $(p<0.001)$. Serum VEGF level significantly increased 7 days after balloon injury both in angioplasty group and BA group. The increase range in BA with angioplasty group was higher than that of the angioplasty group $(p<0.01)$. The serum VEGF level 7 days after injury had linearly negative correlation with SI. In the second experiment, arterial neointima hyperplasia reached delsummit at 28 days in the angioplasty group and 14 days in the BA with angioplasty group. Neointimal and media area of BA with angioplasty at different times (14th, 28th, 45thday) were significantly decreased compared with angioplasty group $(p<0.001)$. The rate of PCNA positive cell increased statistically in BA with angioplasty group than in angioplasty group at 14 th day $(p<0.01)$. The rat of $\alpha$-actin positive cell increased significantly in BA with angioplasty group compared with angioplasty group $(\mathrm{p}<0.01)$ at 14 th day.

Conclusions Bosentan may be effective on artery restenosis by inhibiting neointimal hyperplasia, increasing the serum VEGF level, reducing the proliferation, migration and transconformation of vascular smooth muscle cells.

Note: Combination of paper e0367 and e0371.

\section{e0051 MIR214 IS UPREGULATED DURING VENTRICULAR REMODELLING POST MI}

doi:10.1136/hrt.2010.208967.51

Bing Shi, Lin Mi, Haiyi Yu, Wei Gao. Peking University Third Hospital

Objective MicroRNAs (miRNAs) are endogeneous, single-stranded non-coding RNA molecules about 22 nucleotides long, del regulating target gene expression post-transcriptionally by base pairing with specific binding sites located in the 3' - untranslated regions (UTRs) of downstream target mRNAs. miRNAs play important roles in the regulation of a multitude of physiological functions such as cell differentiation, proliferation, apoptosis and immune response. Recent studies suggest that aberrant expression of miRNAs is associated with cardiovascular diseases. Those miRNAs exhibit unique spatial expression patterns that might become biomarker of diagnosis and target of treatment of ventricular remodelling. In present study, the expression level of miR-214 during ventricular remodelling post MI was detected.

Methods Rats underwent left descending coronary ligation or sham surgery. Rats with MI was assigned to two groups $(n=5)$. Realtime PCR was developed to detect the expression of miR-214 in myocardium and plasma.

Results The expression level of miR-214 in both myocardium and plasma were up-regulated in the 14 th and 28 th day post MI. Compared to sham group, the expression level of miR-214 in myocardium increased by $33 \%(1.33 \pm 0.12$ vs $1.00 \pm 0.02, p<0.01)$ in the 14 th day and by $88 \%(1.88 \pm 0.08$ vs $1.00 \pm 0.02, p<0.01)$ in the 28 th day post MI. Compared to sham group, the expression of miR214 in plasma increased by $60 \%(1.60 \pm 0.09$ vs $1.00 \pm 0.06, p<0.01)$ in the 14 th day and by $116 \%(2.16 \pm 0.13$ vs $1.00 \pm 0.06, p<0.01)$ in the 28th day post MI.

Conclusions The expression level of miR-214 in myocardium and in plasma up-regulated in the progress of ventricular remodelling post $\mathrm{MI}$ in rat. The dynamic change of miR-214 may potentially become a new biomarker in ventricular remodelling post MI.

\section{e0052 VALSARTAN REVERSED VASCULAR FIBROSIS THROUGH THE BLOCKADE OF THE AT1-MEDIATED TGF- $\beta$ /SMAD SIGNAL PATHWAY IN THE FAT-FED, STREPTOZOTOCIN- TREATED RATS}

doi:10.1136/hrt.2010.208967.52

Sun Hui, Zhang Wei, Zhong Ming, Zhao Yong, Su Guohai, Zhang Yun. The Key Laboratory of Cardiovascular Remodelling And Function Research, Chinese Ministry of Education And Chinese Ministry of Public Health, Shandong University, Qilu Hospital, Ji'nan, China

Objective Angiotensin II (AII) and transforming growth factor- $\beta$ (TGF- $\beta$ ) are closely involved in the pathogenesis of diabetic complications. The aim of this study was to clarify the role of AII in the regulation of the TGF- $\beta$ system in diabetic vascular dysfunction. Methods Male Wistar rats were randomly divided into three groups: normal control, diabetic rats and valsartan group. Diabetes was induced by high-calorie diet for 4 weeks and a single intraperitoneal injection of streptozotocin (STZ) thereafter. The expression of TGF$\beta 1 /$ Smads signalling was analysed by real-time reverse transcriptasePCR and immunohistochemistry in aorta of three groups.

Results Compared with control group, the expression of both TGF- $\beta$ I $(27.4013 \pm 10.49256$ vs $15.1254 \pm 6.64343, p<0.01)$, TGF- $\beta$ receptor types II $(28.0173 \pm 10.22042$ vs $10.0561 \pm 8.22275, \mathrm{p}<0.01)$ and activation of the smad2/3 (31.4029 \pm 10.44721 vs $12.8769 \pm 6.98547$, $\mathrm{p}<0.001)$ signalling pathway were up-regulated in the vasculature in diabetic rats. Compared with diabetic group, active TGF- $\beta$ $(18.5682 \pm 10.29359$ vs $27.4013 \pm 10.49256, \mathrm{p}<0.05)$ and $S m a d 2 / 3$ $(20.5209 \pm 7.82756$ vs $31.4029 \pm 10.44721, \mathrm{p}<0.01)$ protein levels were reduced in the aorta after the treatment of valsartan.

Conclusions Our results suggest that AT1 receptor antagonist has reversed vascular fibrosis through the blockade of the AT1-mediated TGF- $\beta /$ Smad signal pathway in the diabetic rats with vascular dysfunction. These observations may del support additional, beneficial effects of angiotensin receptor antagonists observed during del diabetic vascular complications.

Note: $\mathrm{n}=$ ? to be sent back.

\section{e0053 ELECTROPORATION-MEDIATED ANGIOTENSIN II TYPE 2 RECEPTOR GENE TRANSFECTED INTO RAT CAROTID ARTERIES AND THE EFFECTS OF AT2R GENE TRANSFER ON NEOINTIMAL HYPERPLASIA IN RAT CAROTID ARTERIES AFTER BALLOON ANGIOPLASTY}

doi:10.1136/hrt.2010.208967.53

Defeng Liu, Jianping Liu.

Aim To study the effects of Electroporation on the angiotensin II type 2 receptor (AT2R) transfected into rat carotid arteries and study the effects of AT2R gene transfer on neointimal hyperplasia in rat carotid arteries after balloon angioplasty.

Methods Electroporation-mediated AT2R gene transfected into rat carotid arteries after the establishment of rat carotid balloon injury restenosis model. The arteries were harvested at 5 days, 14 days and 21 days after gene transfer. The expression of AT2R in arteries and morphology analysis were evaluated by fluorescence microscope, immunohistochemistry, HE staining and in situ hybridisation.

Results Electroporation-mediated AT2R gene delivered into injured rat carotid arteries significantly up-regulated the levels of AT2R 
mRNA in neointima from day 5 to day 14 . At day 21, compared with no Electroporation-mediated group, no transfection group and GFP transfection group, AT2R transfection reduced $\mathrm{I} / \mathrm{M}$ intimal/medial area ratio significantly $(0.85 \pm 0.1,1.32 \pm 0.19,1.51 \pm 0.19,1.49 \pm 0.25$, $\mathrm{p}<0.01)$. No significant difference between no Electroporationmediated group, GFP group and no transfection group was observed. Conclusion The results of this study provide evidence that electroporation is an effective means for introducing naked AT2R DNA into the blood vessel wall and gene transfer of AT2R in vessel wall may effectively inhibit VSMC proliferation and neointimal hyperplasia in the rat carotid arteries after balloon angioplasty.

\section{e0054 RECOMBINANT ADENO-ASSOCIATED VIRUS SEROTYPE 9 TRANSFECTION OF RATS H9C2 CELLS IN VITRO}

doi:10.1136/hrt.2010.208967.54

Gao Xia, Ma Yi-Tong, Yang Yi-Ning, Xiang Yang, Chen Bang-Dang, Liu Fen. Department of Cardiology, The First Affiliated Hospital of Xinjiang Medical University

Objective To evaluate del transfection efficiency using recombinant adeno-associated virus Serotype 9 mediated enhanced green fluorescent protein (rAAV9- EGFP) to rats $\mathrm{H} 9 \mathrm{C} 2$ cells and the impact on growth of H9C2 cells.

Methods rAAV9-EGFP was transfected into H9C2 cells at different multiplicities of infection $(\mathrm{MOI}=1 \times 105,1 \times 106,1 \times 107)$. EGFP expression in the cells was observed under inverted fluorescence microscope and the EGFP-positive cell percentage determined by flow cytometry. Alamar Blue assay was used to assess the proliferation of the transfected cells.

Results The cells with rAAV9-EGFP transfection at MOI of $1 \times 106$ and $1 \times 107$ began to exhibit EGFP expression 1 days del after transfection and the cells transfection at MOI of $1 \times 105$ began to exhibit EGFP expression 2 days after transfection. The fluorescence intensity increased with the MOI used for transfection. EGFP expression reached the maximum on day 4 , at the point of which the transduction efficiency of rAAV9-EGFP in H9C2 cells was $(14.1 \pm 0.2) \%, \quad(35.1 \pm 4.8) \%$ and $(56.8 \pm 0.1) \%$. Corresponding to MOIs of $1 \times 105,1 \times 106$ and $1 \times 107$, respectively. Alamar Blue assay did not reveal significant difference in the absorbance between the transfected cells and the control cells after transfection.

Conclusions rAAV9-EGFP gene can be del transfected in a stable manner and efficiently expressed in H9C2 cells without causing cell growth inhibition. This del The results of this study played foundation (del) (provides a platform) for further research.

\section{e0055 RELATIONSHIP BETWEEN GENETIC POLYMORPHISM OF CYTOCHROME P4502C19 AND CORONARY ARTERY DISEASE IN XINJIANG UIGUR POPULATION}

doi:10.1136/hrt.2010.208967.55

Yang Yining, Ma Yitong, Wang Xinlei, Liu Fen, Xiang Xie. Department of Cardiology, The First Affiliated Hospital Xinjiang Medical University

Objective To study the relationship between cytochrome P450 family 2C19 subfamily genetic polymorphism and coronary artery disease in Xinjiang Uigur Population.

Methods There are (del) $370 \mathrm{CAD}$ patients and 190 control subjects, who (del) were tested for the genotypes for the (of) CYP2C19 SNPs rs4986893 with the methods (use) of PCR and analysed by restriction fragment length polymorphism. Blood press, (del) (,) plasma biochemical results and smoking were also included (del) (assessed). Statistic analyses were (del) (analysation was) performed with the SPSS 12.0 software package. The Hardy-Weinberg equilibrium was tested in all the groups.
Results Uigur, the genotypes distribution of the control group and the CHD group were in the Hardy-Weinberg equilibrium the (del), $\mathrm{p}>0.05$ (for case $\mathrm{p}=0.2$, for control $\mathrm{p}=0.068$ ). The frequency of $\mathrm{AA}$, AG and GG were $0.3 \%, 0.5 \%$ and $94.3 \%$ in the CAD group, compared with the control group that were $0.6 \%, 5.6 \%$ and $93.8 \%$ respectively. For $\mathrm{x}^{2}=0.107$, the $\mathrm{p}$ value was 0.743 . There was no significant difference in between the two groups. But the (amt?level???) blood glucose in the two groups had a difference (del). Smoking was also different in the CAD and the control groups $(p=0.0002)$.

Conclusions The distribution frequency of the CYP2C19 SNPs rs4986893 genotypes in the Uigur population with or without CAS is not significantly different with the blood glucose and smoking both have correlation with $\mathrm{CAD}$ (del).

\section{e0056 CORRELATION BETWEEN VIABLE MYOCARDIUM AND PZF IN SWINE MODEL OF ACUTE MYOCARDIAL INFARCTION}

doi:10.1136/hrt.2010.208967.56

Lin Tao, Ma Yi-Tong, Yang Yi-Ning, Mu Hu-Yati. Department of Cardiology, The First Affiliated Hospital, Xinjiang Medical University

Objective The aim of this study is to explore the relationship between the viable myocardium and Pzf of related coronary artery in swine model of acute myocardial.

Methods 13 swines, received coronary arteriongraphy after anaesthetic tracheal cannula, the pressure of coronary arteries were measured through guiding wire and the distal flow velocity of left anterior descending coronary arteries measured through doppler wire. Drawing the pressure- flow velocity coordinate graph, then calculated Pzf. Blocked up distal of left anterior descending coronary arteries by angioplasty balloon for 60-90 min to establish the model of acute myocardial infarction. The Pzf were calculated repeadly. Analysis the Pzf difference between before and after the model of acute myocardial infarction established. Taken out of those swines' hearts after executed them, sliced the cardiac muscle to dye with TTc to detect the viable myocardium, then cutted them from the left ventricular myocardium. The percent value of area viable myocardium were estimated by calculated the ratio of weight of viable myocardium to whole left venricular myocardium Drawing the percent value of area viable myocardium-Pzf after the model of acute myocardial infarction established coordinate graph and observed relationship between them.

Results 11 out of 13 swines were successfully established models of acute myocardial infarction by coronary occlusion. 2 swines died of entricular fibrillation after their coronaries were occluded 45 and 65 min respectively. The viable myocardium could be detected by using TTc dyeing. The result of statistics analysis confirmed that the percent value of area viable myocardium were negative correlation with Pzf. Conclusion The swine model of acute myocardial infarction can be established successfully by coronary occlusion with angioplasty balloon. The percent value of area viable myocardium were negative correlation with Pzf, which showed that Pzf was a new method to predict the area of viable myocardium after acute myocardial infarction.

\section{e0057 CORRELATION OF ABCA1 GENE R219K IN THE UIGHUR NATIONAL MINORITY IN XINJIANG WITH LIPIDMETABOLISM AND THE RISK OF MI}

doi:10.1136/hrt.2010.208967.57

Sun Minghui, Ma Yitong, Yang Yining. Department of Cardiology, The First Affiliated Hospital, Xinjiang Medical University

Objective To study the correlation of ATP binding cassette transporter $\mathrm{A} 1$ (ABCA1) and the gene polymorphism of $\mathrm{R} 219 \mathrm{~K}$ in the 